Check for updates

Cite this: Mater. Chem. Front., $2019,3,620$

Received 10th December 2018 Accepted 29th January 2019

DOI: $10.1039 / c 8 q m 00633 d$

rsc.li/frontiers-materials

\title{
An ESIPT-based fluorescent switch with AIEE, solvatochromism, mechanochromism and photochromism $\dagger$
}

\author{
Xuedan Liu, ${ }^{a}$ Aisen Li, ${ }^{b}$ Weiqing $\mathrm{Xu}$, (D) $^{\mathrm{b}}$ Zhiyong Ma (D) *a and Xinru Jia (D) ${ }^{\mathrm{c}}$
}

\begin{abstract}
In this study, we report a newly synthesized molecule 1 via linking a benzothiazole to rhodamine, which could exhibit AIEE, unique acidichromism and solvatochromism in solution, fascinating photochromism in a polymer matrix, intriguing mechanochromism in solid state and remarkable piezochromism in the crystal. $\mathbf{1}$ is a rarely reported organic molecule, which is sensitive to multiple stimuli such as acid/base, force, pressure, light and solvent polarity due to its distinct intramolecular hydrogen bonding and spirolactam structure. 1 demonstrated AIEE property in a $\mathrm{THF} / \mathrm{H}_{2} \mathrm{O}$ system because of the water-induced aggregation and aggregation-suppressed nonradiative relaxation of the excited states. In the solution, 1 afforded yellowish-green emission at $520 \mathrm{~nm}$ (keto emission) in a non-polar solvent and blue emission at $447 \mathrm{~nm}$ (enol emission) in a polar solvent. Surprisingly, a blue shift in the emission peak was observed in a DCM solution upon treatment with acid or base, which is quite different from that of traditional rhodamine. In the solid state, the emission color of 1 changed from yellowish-green to red upon grinding or applying hydrostatic pressure, owing to the force-induced ring-opening reaction. In the polymer matrix, 1 manifested reversible photochromic behaviour and could serve as a good photoprinting material. This study is expected to contribute to the development of multistimuli-responsive fluorescent switch with multiple functions in different states.
\end{abstract}

\section{Introduction}

Fluorescent molecular switches with tunable luminescent behavior using external stimuli, ${ }^{1-10}$ especially in their solid state, ${ }^{11-14}$ have attracted considerable attention due to their potential applications in optical data recording, displays, chemical sensors and fluorescent probes. ${ }^{15-29}$ Rhodamines and their derivatives are well known as molecular switches and have been widely studied in intelligent materials, owing to the remarkable absorption and emission change that occurs during the reversible isomerization between the closed-ring and open-ring forms. ${ }^{30,31}$ However, aggregation-induced quenching of rhodamines limits

\footnotetext{
${ }^{a}$ Beijing State Key Laboratory of Organic-Inorganic Composites, College of Chemical Engineering, Beijing University of Chemical Technology, Beijing 100029, China. E-mail: mazhy@mail.buct.edu.cn

${ }^{b}$ State Key Laboratory for Supramolecular Structure and Materials, Institute of Theoretical Chemistry, Jilin University, Changchun 130012, China ${ }^{c}$ Beijing National Laboratory for Molecular Sciences, Key Laboratory of Polymer Chemistry and Physics of the Ministry of Education, College of Chemistry and Molecular Engineering, Peking University, Beijing 100871, China

$\dagger$ Electronic supplementary information (ESI) available: Details of experimental sections, including synthesis, characterization, spectra, SAXSS patterns and optical images. CCDC 1883880. For ESI and crystallographic data in CIF or other electronic format see DOI: 10.1039/c8qm00633d
}

its applications in the solid state. Highly emissive organic fluorescent materials with multistimuli-responsive behavior in different states are in high demand. Therefore, it is of great significance to fabricate sensor materials with multiple functions to further widen the scope of application in different fields. ${ }^{32-37}$ However, reports on such materials are very rare.

The photo-induced proton transfer through an intramolecular hydrogen bond is defined as excited-state intramolecular proton transfer (ESIPT). ${ }^{38}$ Organic ESIPT-based fluorescent molecules have been objects of interest in the past decades because they exhibit remarkably large Stokes shift, unique dual emission and special sensitivity to the external microenvironment due to their four-level photophysical cycles. ${ }^{39}$ Because of the transient changes involved in the ESIPT process, ESIPT emission is quite easily affected by external stimuli, resulting in the change of fluorescence properties. ${ }^{40-44}$ Therefore, making good use of the ESIPT process is an effective way to fabricate smart luminescent materials that are sensitive to external stimuli such as solvent polarity, pH, heat, mechanical force and pressure. ${ }^{45-50}$ The most studied ESIPT system consists of the derivatives of 2-(2'-hydroxyphenyl)benzimidazole (HBI), 2-(2'-hydroxyphenyl)benzoxazole (HBO) and 2-( $2^{\prime}$-hydroxyphenyl)benzothiazole (HBT), where the hydroxyl linked to its conjugated group acts as the proton donor and the imidazole/oxazole/thiazole ring acts as 


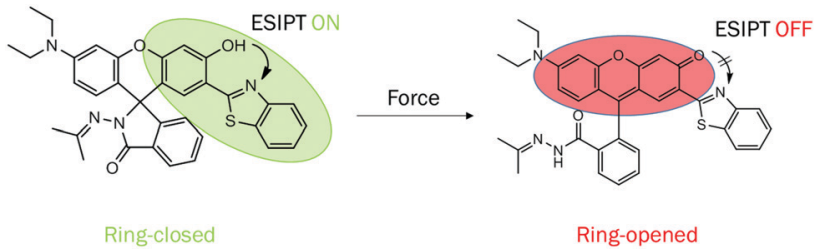

Scheme 1 Molecular structures of the target molecule 1. The ring-closed form undergoes ring opening on application of force.

the proton acceptor. ${ }^{51}$ Hence, the combination of rhodamine with ESIPT is predicted to yield a new type of multi-responsive luminescent material both in solution and solid state. Prof. Xiao-Feng Yang has reported a benzothiazole-substituted rhodamine, which exhibited ESIPT and ratiometric response towards copper ion. ${ }^{52}$ However, the study neglected the responses to other stimuli and the fluorescent properties in the solid state.

In this study, we report the synthesis of a new molecule $\mathbf{1}$ (Scheme 1) by linking a benzothiazole to rhodamine, which is shown to exhibit AIEE, unique acidichromism and solvatochromism in solution, fascinating photochromism in the polymer matrix, intriguing mechanochromism in solid state and remarkable piezochromism in the crystal due to its distinct intramolecular hydrogen bonding and spiro-lactam structure. The single crystal was developed successfully, which showed an antiparallel slip-stacked dimer structure. 1 showed AIEE property in the $\mathrm{THF} / \mathrm{H}_{2} \mathrm{O}$ system because of the water-induced aggregation and aggregation-suppressed nonradiative relaxation ways of the excited states. In solution, 1 exhibited a yellowish-green emission at $520 \mathrm{~nm}$ (keto emission) in the non-polar solvent and blue emission at $447 \mathrm{~nm}$ (enol emission) in the polar solvent in general. Surprisingly, a blue shift and enhancement of emission were observed in the DCM solution upon treatment with acid or base, which was quite different from that of traditional rhodamine. In the solid state, on grinding or applying hydrostatic pressure, the emission color of $\mathbf{1}$ changed from yellowishgreen to red owing to the force-induced ring-opening reaction. In the polymer matrix, $\mathbf{1}$ manifested a reversible photochromic behaviour and could serve as a good photo-printing material. To the best of our knowledge, $\mathbf{1}$ is a rarely reported organic molecule that is sensitive to multiple stimuli such as acid/base, force, pressure, light and solvent polarity. This study would contribute to the development of a multistimuli-responsive fluorescent switch with multiple functions in different states.

\section{Results and discussion}

Compound 2 was acquired from 2,4-dihydroxybenzaldehyde and 2-aminobenzenethiol by a typical two-step condensation reaction, according to the literature. ${ }^{52}$ The target molecule 1 was obtained via Schiff base reaction between compound 2 and acetone (white powder, $53 \%$ yield). ${ }^{1} \mathrm{H}$ NMR, ${ }^{13} \mathrm{C}$ NMR, HR-MS and crystallographic analysis were performed to verify the molecular structures and product purity. The detailed synthetic procedures and characterization processes are supplied in the ESI $\dagger$ (Scheme S1 and Fig. S1-S3).
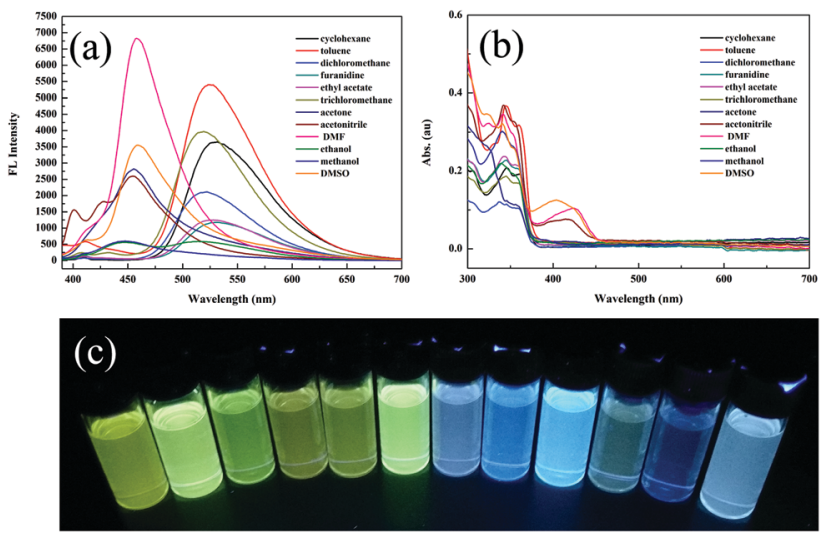

Fig. 1 (a) Fluorescence spectra, (b) absorption spectra and (c) optical images of 1 in different organic solvents $\left(20 \mu \mathrm{M}, \lambda_{\mathrm{ex}}=365 \mathrm{~nm}\right.$, from left to right: cyclohexane, toluene, DCM, THF, ethyl acetate, chloroform, acetone, acetonitrile, DMF, ethanol, methanol, DMSO).

Firstly, we studied the photophysical properties of $\mathbf{1}$ in different organic solvents. Interestingly, 1 showed unique sensitivity to different polarities of the solvents. For example, in nonpolar solvents such as cyclohexane and toluene, the emission peak of 1 was centered around $520 \mathrm{~nm}$ due to ESIPT facilitated by the strong intramolecular hydrogen bonding (Fig. 1a and c). With increasing polarity, another emission peak was detected at $447 \mathrm{~nm}$ in acetone and methanol, which was ascribed to the excited states of the enol form. It is worth mentioning that a dual-fluorescence was observed in ethanol, which suggested that both enol and keto forms coexisted in the excited states. Unexpectedly, a new emission band appeared at $457 \mathrm{~nm}$ only in DMSO, DMF and acetonitrile solvents. Correspondingly a new broad absorption band was located in the range of 375-450 $\mathrm{nm}$ in the absorption spectra (Fig. 1b), which was attributed to the phenolic anion generated via deprotonation induced by the intermolecular hydrogen bonding between the phenolic hydroxyl groups and the solvent molecules as reported previously. ${ }^{53}$ Thus, 1 remarkably exhibited triple emissions with varying solvent polarity.

The aggregation-induced emission enhancement (AIEE) property of 1 was studied in the THF $/ \mathrm{H}_{2} \mathrm{O}$ system. Interestingly, 1 exhibited AIEE behavior though it did not contain typical AIEE structures such as the TPE moiety. In the diluted THF solution of $\mathbf{1}$, weak emission bands were detected at $405 \mathrm{~nm}$ and $430 \mathrm{~nm}$ (Fig. 2a and c), which were attributed to the xanthene moiety. The typical emission band ascribed to ESIPT was of low intensity with a low quantum yield of $2.78 \%$ due to the free rotation of thiazole ring. The fluorescent behavior of 1 changed with the water fraction in the system. The target molecule $\mathbf{1}$ began to aggregate and a yellow emission centered at $520 \mathrm{~nm}$ emerged when the water proportion $\left(f_{\mathrm{w}}\right)$ reached $70 \%$. The emissive intensity increased continuously with the water proportion. When $f_{\mathrm{w}}$ was above $90 \%, 1$ was highly emissive at $520 \mathrm{~nm}$ with a high quantum yield of $20.79 \%$ because the aggregation of 1 molecules prohibited the nonradiative relaxation channels and facilitated the ESIPT process. With increasing water proportion, the absorption bands red-shifted on account of Mie scattering 

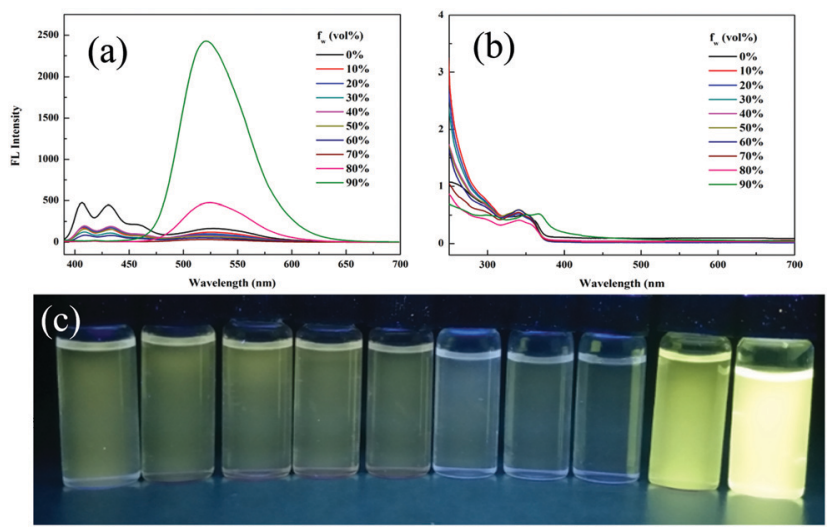

Fig. 2 (a) Fluorescence spectra, (b) absorption spectra and (c) optical images of 1 in different THF/water $(v / v)$ mixtures $\left(20 \mu \mathrm{M}, \lambda_{\text {ex }}=365 \mathrm{~nm}\right)$.

effect (Fig. 2b), which further verified the aggregation process of $\mathbf{1}$. These results reveal that $\mathbf{1}$ is AIEE active.

The reversible ring-opening/closing isomerization of 1 triggered by acid and base was investigated in dichloromethane (DCM) solution. In contrast to rhodamine lactam, the fluorescence spectra of the fluorescent switch 1 showed a blue shift upon stimulation by acid or base. In the original DCM solution, the emission peak was located at $520 \mathrm{~nm}$ due to ESIPT. On addition of $0.01 \mathrm{M} \mathrm{NaOH}$ ethanol solution, the DCM solution turned red under visible light and changed to bright blue under $365 \mathrm{~nm}$ UV light, unexpectedly. Two new absorption bands at $385 \mathrm{~nm}$ and $565 \mathrm{~nm}$ were detected (Fig. 3a and Fig. S4, ESI $†$ ), which confirmed the deprotonation process and the ringopening reaction. The new emission peak intensity at $457 \mathrm{~nm}$ gradually strengthened with the addition of base, which was quite similar to that in DMSO and provided solid evidence that it corresponded to the phenolic anion. As for the acid stimulus, the DCM solution turned red under visible light and the emission red-shifted from $520 \mathrm{~nm}$ to $477 \mathrm{~nm}$ and further to $415 \mathrm{~nm}$ with an enhancement in the intensity on increasing the volume of TFA (Fig. 3b and Fig. S5, ESI $\dagger$ ). Three new absorption bands were detected at $370 \mathrm{~nm}, 508 \mathrm{~nm}$ and $546 \mathrm{~nm}$ (Fig. S5, ESI $\dagger$ ), thus indicating the occurrence of the ring-opening reaction. In acidic condition, the $477 \mathrm{~nm}$ and $415 \mathrm{~nm}$ emissions could probably be ascribed to the enol forms. It is worth mentioning that this is the first rhodamine derivative to show both emission blue shift and emission enhancement when induced with an acid or base.
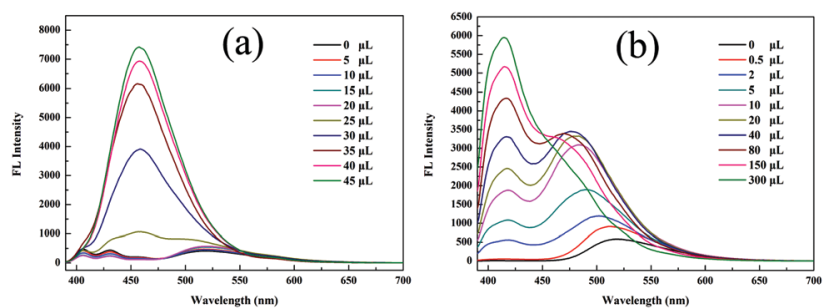

Fig. 3 Fluorescence spectra of 1 after adding (a) $0.01 \mathrm{M} \mathrm{NaOH}$ ethanol solution and (b) TFA.
Tuning the fluorescent behavior of solid-state materials through external stimuli has been in the limelight in recent years. 1 displayed reversible mechanochromic behavior in the solid state. The pristine powder was obtained after removing the solvent completely. Initially, it showed an emission peak at $520 \mathrm{~nm}$ with yellowish-green color (QY: $46.12 \%, \tau=4.27 \mathrm{~ns}$ ) (Fig. 4a) due to ESIPT. Even after slight shearing, the emission peak at $520 \mathrm{~nm}$ was retained and no shift was observed. We conducted XRD and DSC to gain insight into the microstructural changes after applying force. For the pristine powder, numerous sharp scattering peaks were detected, indicating that the pristine powder was highly crystalline. After slight shearing, the scattering peaks disappeared (Fig. 4c), implying a force-induced phase transition from ordered stacking to an amorphous state. In the DSC curves, there were two sharp endothermic peaks at $172{ }^{\circ} \mathrm{C}$ (assigned to the solid-to-solid transition) and $258{ }^{\circ} \mathrm{C}$ (melting point) for the pristine powder (Fig. S6, ESI $\dagger$ ); however, only a glass transition process was observed for the ground powder, verifying that the crystal structure was damaged by external force. The above results disclosed that the ESIPT-based emission was independent of the physical molecular stacking and relied totally on the strong intramolecular hydrogen bonding in this system. Further shearing endowed the powder with a reddish emission at $600 \mathrm{~nm}$, which was assigned to the ringopen form of $\mathbf{1}$. We tested the solid-state absorption spectra of the ground red powder, which showed a broad absorption band at $590 \mathrm{~nm}$ (Fig. S7, ESI $\dagger$ ), thus verifying the ring-open form of compound 1. After heavy grinding, the QY decreased drastically to $10.12 \%$ and the lifetime was $2.08 \mathrm{~ns}$, which implied that the ESIPT process was prohibited by the shearing force. After annealing, the reddish color powder regained the original yellowish-green color.
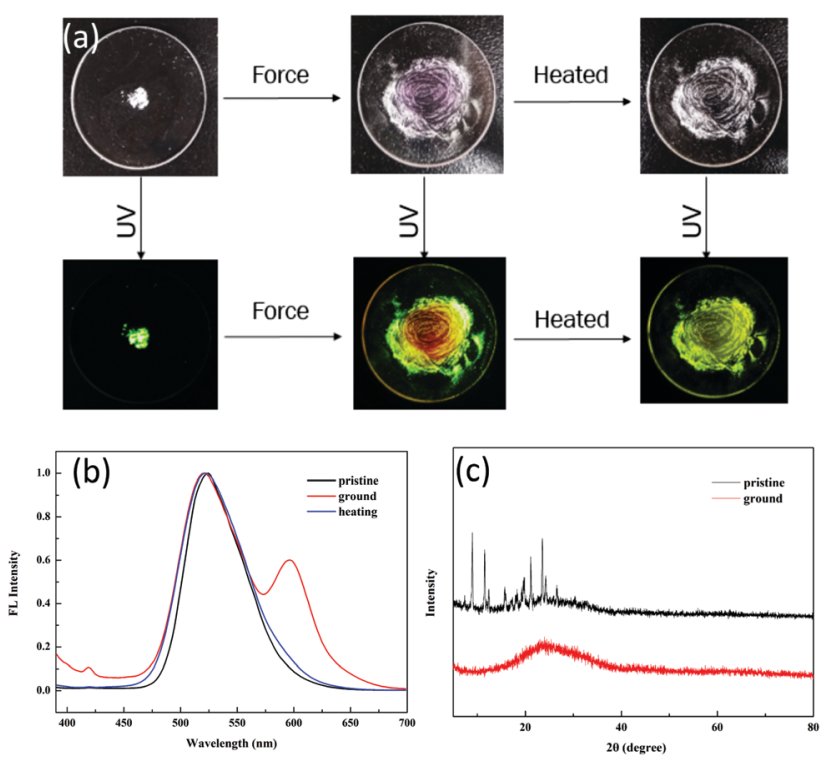

Fig. 4 (a) Optical images of the color-changing process of 1 due to shearing and heating under room light and 365 UV light. (b) Fluorescence spectra of the pristine powder, powder after heavy grinding and the heated powder. (c) XRD patterns of the pristine powder and powder after slight grinding. 
In addition, no scattering peaks was observed in the XRD spectra (Fig. S8, ESI $\dagger$ ), suggesting that the ring-closed molecule structure of 1 could be recovered by heating but the ground sample could not regain the original crystal state by heating.

In order to dynamically monitor the color-changing process in situ and quantify the relationship between the emission transformation and the external force, single crystals of 1 were subjected to hydrostatic pressure in a DAC equipment, where silicone oil was used as the pressure transmitting medium. Notably, it exhibited a clear color change when a pressure was applied. The original single crystal had bright green emission at $520 \mathrm{~nm}$ under $365 \mathrm{~nm}$ UV light due to the ESIPT process. When the pressure was increased to $10.08 \mathrm{GPa}$, the single crystal turned dark-red with an emission peak at $590 \mathrm{~nm}$ (Fig. 5a and b), which was due to the pressure-induced ring-opening reaction of the spiro-lactam. At the same time, the emission wavelength redshifted gradually (Fig. S9, ESI $\dagger$ ) and the fluorescence intensity weakened correspondingly. Once the pressure was released, the single crystal could be restored to its original green color, indicating that the ring-open species of 1 recovered to the ringclosed form (Fig. 5c). Hence, the pressure-responsive emission color change was completely reversible.

To further explore the interdependence of the molecular structure and spectroscopic behavior, and gain insights into the mechanochromic mechanism, the single crystal structure of $\mathbf{1}$ was analyzed (Table S1, ESI $\dagger$ ). Interestingly, the single crystals of 1 were obtained accidentally during a trial to cultivate single crystals of 2 in acetone. The single crystal belongs to the monoclinic crystal system with the space group of $P 21 / c$ (No. 14).
Each unit cell contained four molecules (Fig. S10, ESI $\dagger$ ). The rhodamine moiety was highly twisted due to the spiro-carbon atom. The HBT unit and the phenol part were located almost in the same plane with a small dihedral angle of $7.77^{\circ}$ (Fig. 6a), owing to the strong intramolecular hydrogen bonding between the $-\mathrm{OH}$ group and the $\mathrm{N}$ atom, which provides direct evidence for the ESIPT process. Interestingly, the single crystal of $\mathbf{1}$ adopted an antiparallel slip-stacked dimer structure (3.35 $\AA$ ) with a considerable offset by intermolecular $\pi-\pi$ interactions (Fig. 6b). In the single crystal, one molecule interacted with at least seven adjacent molecules via the $\pi-\pi$ interactions, $\mathrm{C}-\mathrm{H} \cdots \pi$ and $\mathrm{C}-\mathrm{H} \cdots \mathrm{H}-\mathrm{C}$ interactions, which suggests that the molecule is highly constrained in the crystal state. When subjected to high pressure, the pressure would be conducted to the spiro-lactam structure and result in the ring-opening reaction.

Generally, the rhodamine amide can undergo a UV lightpromoted heterolytic bond fissure to form a normal colored zwitterionic architecture. However, its very short lifetime in the ring-open state limits its application as a photochromic molecule. In this system, 1 displayed very fine photochromic property in the matrix. Firstly, we distributed the powder of $\mathbf{1}$ on a piece of filter paper by immersing in the THF solution (Fig. S11, ESI $\dagger$ ). The treated filter paper exhibited fascinating photo-activity with a distinct color change from yellow to red. The fluorescence spectra showed that a new emission peak emerged at $595 \mathrm{~nm}$ after UV irradiation (Fig. S12, ESI $\dagger$ ). Secondly, we prepared a polyurethane film containing 1 in the weight ratio of $1 \%$. The PU film demonstrated reversible light-patterning behaviour and was able to serve as a material for photo-printing. Initially,

(a)
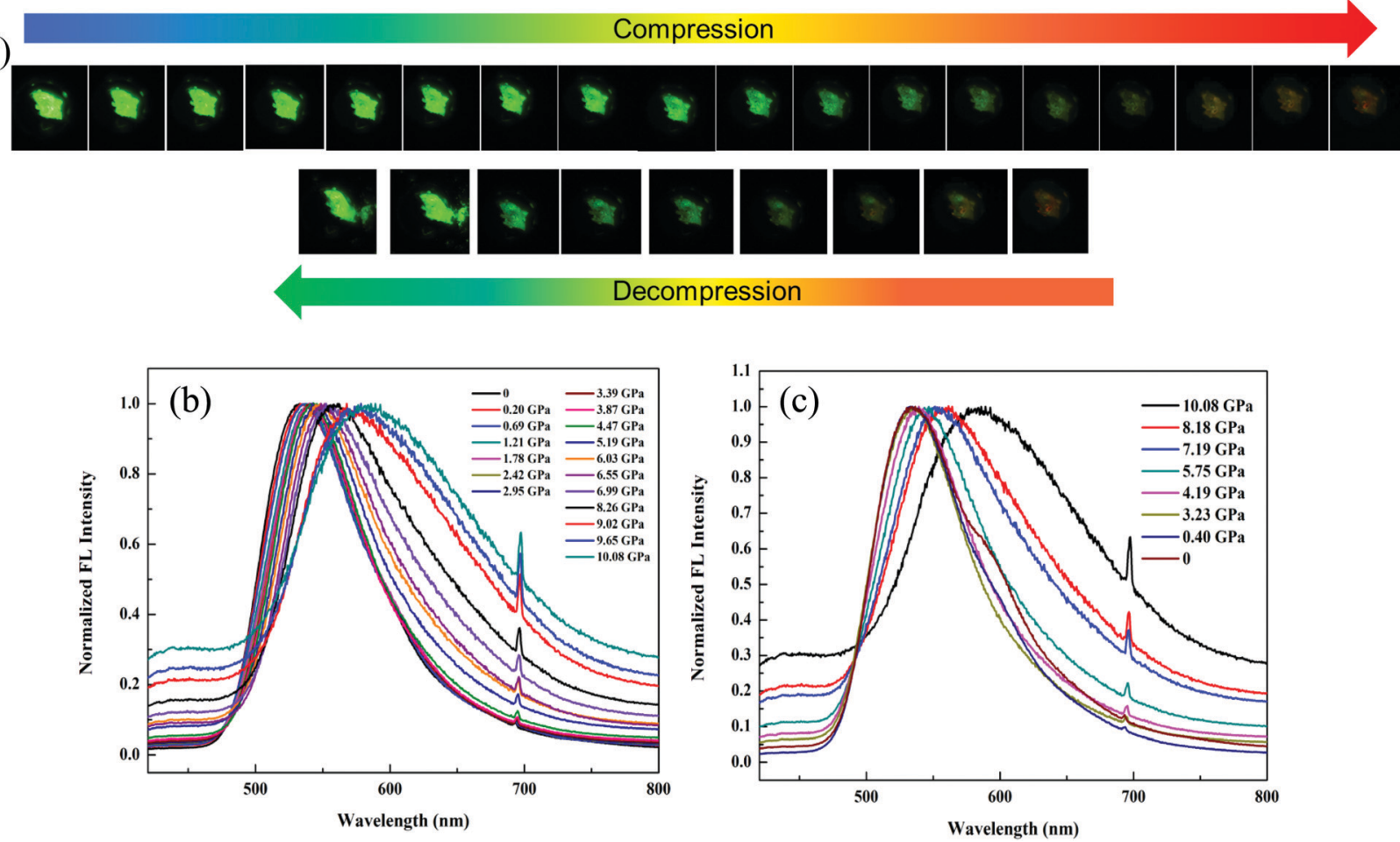

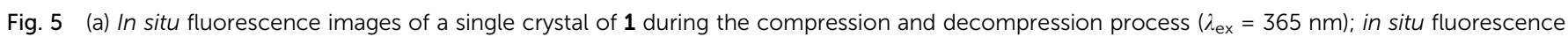
spectra $\left(\lambda_{\mathrm{ex}}=365 \mathrm{~nm}\right)$ during the $(b)$ compression and $(c)$ decompression process $\left(\lambda_{\mathrm{ex}}=365 \mathrm{~nm}\right)$. 

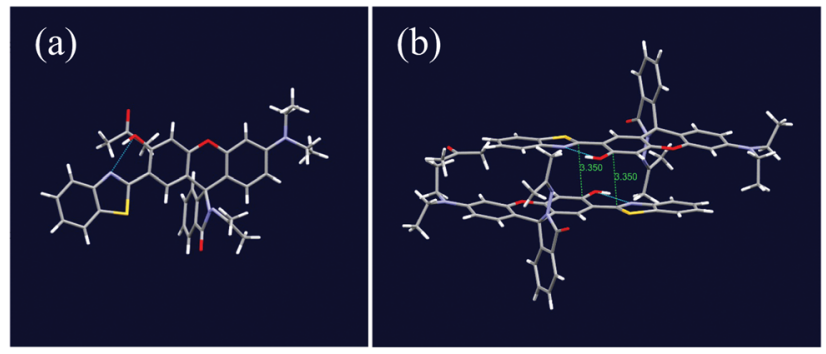

Fig. 6 (a) Monomer and (b) antiparallel slip-stacked dimer unit with close contacts in the single crystal of 1.

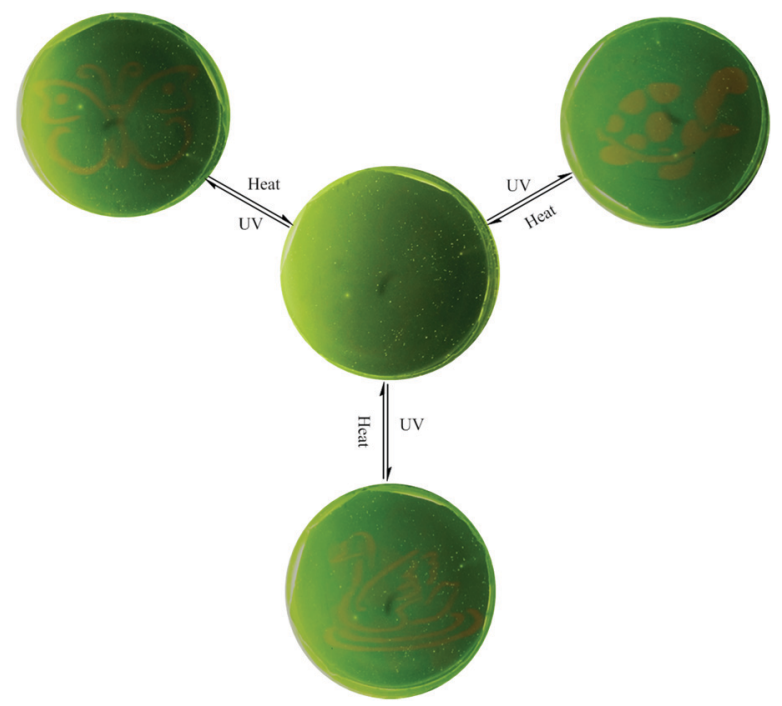

Fig. 7 Three photo-printing cycles of UV light irradiation and heating treatment are demonstrated with three different patterns (butterfly, tortoise and swan) printed on the same PU film (containing $1 \mathrm{wt} \%$ of 1 ).

we covered the PU film with a butterfly pattern and then positioned the film under 365 UV light. As a result, a red butterfly was visualized in the film (Fig. 7). When the film was placed on a hot-plate at $150{ }^{\circ} \mathrm{C}$, the red-colored pattern disappeared without any trace in five minutes. The UV lightinduced emission "green-red" switch could be repeated for multiple cycles, implying fast photo-reversibility and good photostability of 1. After annealing, the film was covered with a new pattern and positioned again under UV light. Consequently, the new pattern appeared clearly, suggesting the easy "writingerasing" nature of the photochromic PU film. This operation could be cycled several times. Fig. 7 shows the pictures of the same film patterned repeatedly through three cycles of UV light irradiation and heating treatment.

\section{Conclusions}

In conclusion, we have reported a new molecule 1 synthesized by fusing rhodamine with HBT, which successfully combines the properties of both molecules and exhibits new properties. $\mathbf{1}$ is endowed with multiple features such as AIEE, acidichromism, solvatochromism, mechanochromism, piezochromism and photochromism, owing to its distinct intramolecular hydrogen bonding and spiro-lactam structure. $\mathbf{1}$ is an unusual organic molecule that responds differently to multiple external stimuli and can lay the foundation for the preparation of smart materials. This study also provides a new strategy to exploit a broad perspective for the development of multistimuli-responsive fluorescent switch.

\section{Conflicts of interest}

There are no conflicts to declare.

\section{Acknowledgements}

This study was financially supported by the National Natural Science Foundation of China (21704002), the Natural Science Foundation of Beijing Municipality (2182054) and the Fundamental Research Funds for the Central Universities (buctrc201723\&ZY1701) to Z. Y. Ma.

\section{Notes and references}

1 Z. Chi, X. Zhang, B. Xu, X. Zhou, C. Ma, Y. Zhang, S. Liu and J. Xu, Chem. Soc. Rev., 2012, 41, 3878-3896.

2 Z. Ma, Z. Wang, M. Teng, Z. Xu and X. Jia, ChemPhysChem, 2015, 16, 1811-1828.

3 A. Lavrenova, D. W. R. Balkenende, Y. Sagara, S. Schrettl, Y. C. Simon and C. Weder, J. Am. Chem. Soc., 2017, 139, 4302-4305.

4 K. Nagura, S. Saito, H. Yusa, H. Yamawaki, H. Fujihisa, H. Sato, Y. Shimoikeda and S. Yamaguchi, J. Am. Chem. Soc., 2013, 135, 10322-10325.

5 Q. Qi, C. Li, X. Liu, S. Jiang, Z. Xu, R. Lee, M. Zhu, B. Xu and W. Tian, J. Am. Chem. Soc., 2017, 139, 16036-16039.

6 T. Seki, N. Tokodai, S. Omagari, T. Nakanishi, Y. Hasegawa, T. Iwasa, T. Taketsugu and H. Ito, J. Am. Chem. Soc., 2017, 139, 6514-6517.

7 M. Tanioka, S. Kamino, A. Muranaka, Y. Ooyama, H. Ota, Y. Shirasaki, J. Horigome, M. Ueda, M. Uchiyama, D. Sawada and S. Enomoto, J. Am. Chem. Soc., 2015, 137, 6436-6439.

8 Y. Sagara and T. Kato, Nat. Chem., 2009, 1, 605-610.

9 L. Huang, C. Wu, L. Zhang, Z. Ma and X. Jia, ACS Appl. Mater. Interfaces, 2018, 10, 34475-34484.

10 C. Wang and Z. Li, Mater. Chem. Front., 2017, 1, 2174-2194.

11 Z. Ma, X. Meng, Y. Ji, A. Li, G. Qi, W. Xu, B. Zou, Y. Ma, G.-C. Kuang and X. Jia, Dyes Pigm., 2019, 162, 136-144.

12 A. Li, Z. Ma, J. Wu, P. Li, H. Wang, Y. Geng, S. Xu, B. Yang, H. Zhang, H. Cui and W. Xu, Adv. Opt. Mater., 2018, 6, 1700647.

13 Y. Liu, Q. Zeng, B. Zou, Y. Liu, B. Xu and W. Tian, Angew. Chem., Int. Ed., 2018, 57, 15670-15674.

14 Y.-B. Gong, P. Zhang, Y.-r. Gu, J.-Q. Wang, M.-M. Han, C. Chen, X.-J. Zhan, Z.-L. Xie, B. Zou, Q. Peng, Z.-G. Chi and Z. Li, Adv. Opt. Mater., 2018, 6, 1800198. 
15 J. Xiong, K. Wang, Z. Yao, B. Zou, J. Xu and X.-H. Bu, ACS Appl. Mater. Interfaces, 2018, 10, 5819-5827.

16 K. Dojin, K. J. Eon and P. S. Young, Adv. Funct. Mater., 2018, 28, 1706213.

17 S. K. Park, I. Cho, J. Gierschner, J. H. Kim, J. H. Kim, J. E. Kwon, O. K. Kwon, D. R. Whang, J.-H. Park, B.-K. An and S. Y. Park, Angew. Chem., Int. Ed., 2016, 55, 203-207.

18 H.-J. Kim, D. R. Whang, J. Gierschner, C. H. Lee and S. Y. Park, Angew. Chem., Int. Ed., 2015, 54, 4330-4333.

19 N. Mizoshita, T. Tani and S. Inagaki, Adv. Mater., 2012, 24, 3350-3355.

20 M. Martínez-Abadía, R. Giménez and M. B. Ros, Adv. Mater., 2018, 30, 1704161.

21 Y. Sagara, S. Yamane, M. Mitani, C. Weder and T. Kato, Adv. Mater., 2016, 28, 1073-1095.

22 A. Julià-López, J. Hernando, D. Ruiz-Molina, P. GonzálezMonje, J. Sedó and C. Roscini, Angew. Chem., Int. Ed., 2016, 55, 15044-15048.

23 Z. Ma, M. Teng, Z. Wang, S. Yang and X. Jia, Angew. Chem., Int. Ed., 2013, 52, 12268-12272.

24 Z. Ma, Z. Wang, X. Meng, Z. Ma, Z. Xu, Y. Ma and X. Jia, Angew. Chem., Int. Ed., 2016, 55, 519-522.

25 Z. Mao, Z. Yang, Y. Mu, Y. Zhang, Y.-F. Wang, Z. Chi, C.-C. Lo, S. Liu, A. Lien and J. Xu, Angew. Chem., Int. Ed., 2015, 54, 6270-6273.

26 J. Yang, Z. Ren, Z. Xie, Y. Liu, C. Wang, Y. Xie, Q. Peng, B. Xu, W. Tian, F. Zhang, Z. Chi, Q. Li and Z. Li, Angew. Chem., Int. Ed., 2017, 56, 880-884.

27 J.-A. Li, J. Zhou, Z. Mao, Z. Xie, Z. Yang, B. Xu, C. Liu, X. Chen, D. Ren, H. Pan, G. Shi, Y. Zhang and Z. Chi, Angew. Chem., Int. Ed., 2018, 57, 6449-6453.

28 X. Wu, J. Guo, Y. Cao, J. Zhao, W. Jia, Y. Chen and D. Jia, Chem. Sci., 2018, 9, 5270-5277.

29 Q. Li and Z. Li, Adv. Sci., 2017, 4, 1600484.

30 X. Chen, T. Pradhan, F. Wang, J. S. Kim and J. Yoon, Chem. Rev., 2011, 112, 1910-1956.

31 H. N. Kim, M. H. Lee, H. J. Kim, J. S. Kim and J. Yoon, Chem. Soc. Rev., 2008, 37, 1465-1472.

32 Q. Qi, J. Qian, S. Ma, B. Xu, S. X.-A. Zhang and W. Tian, Chem. - Eur. J., 2015, 21, 1149-1155.

33 X. Su, Y. Ji, W. Pan, S. Chen, Y.-M. Zhang, T. Lin, L. Liu, M. Li, Y. Liu and S. X.-A. Zhang, J. Mater. Chem. C, 2018, 6, 6940-6948.
34 Q. Yu, X. Su, T. Zhang, Y.-M. Zhang, M. Li, Y. Liu and S. X.-A. Zhang, J. Mater. Chem. C, 2018, 6, 2113-2122.

35 L. Huang, Y. Qiu, C. Wu, Z. Ma, Z. Shen and X. Jia, J. Mater. Chem. C, 2018, 6, 10250-10255.

36 Z. Ma, Y. Ji, Y. Lan, G.-C. Kuang and X. Jia, J. Mater. Chem. C, 2018, 6, 2270-2274.

37 Y. Li, Z. Ma, A. Li, W. Xu, Y. Wang, H. Jiang, K. Wang, Y. Zhao and X. Jia, ACS Appl. Mater. Interfaces, 2017, 9, 8910-8918.

38 A. P. Demchenko, K.-C. Tang and P.-T. Chou, Chem. Soc. Rev., 2013, 42, 1379-1408.

39 J. E. Kwon and S. Y. Park, Adv. Mater., 2011, 23, 3615-3642.

40 F. Wu, L. Wang, H. Tang, D. Cao and X. Jiang, Chem. - Asian J., 2018, 13, 950-954.

41 S. Tsuchiya, K.-i. Sakai, K. Kawano, Y. Nakane, T. Kikuchi and T. Akutagawa, Chem. - Eur. J., 2018, 24, 5868-5875.

42 Y. Niu, R. Wang, P. Shao, Y. Wang and Y. Zhang, Chem. Eur. J., 2018, 24, 16670-16676.

43 T. He, N. Niu, Z. Chen, S. Li, S. Liu and J. Li, Adv. Funct. Mater., 2018, 28, 1706196.

44 F. Borbone, A. Tuzi, B. Panunzi, S. Piotto, S. Concilio, R. Shikler, S. Nabha and R. Centore, Cryst. Growth Des., 2017, 17, 5517-5523.

45 V. S. Padalkar, D. Sakamaki, K. Kuwada, A. Horio, H. Okamoto, N. Tohnai, T. Akutagawa, K.-i. Sakai and S. Seki, Asian J. Org. Chem., 2016, 5, 938-945.

46 V. S. Padalkar, D. Sakamaki, N. Tohnai, T. Akutagawa, K.-i. Sakai and S. Seki, RSC Adv., 2015, 5, 80283-80296.

47 R. Wei, P. Song and A. Tong, J. Phys. Chem. C, 2013, 117, 3467-3474.

48 D. Yao, S. Zhao, J. Guo, Z. Zhang, H. Zhang, Y. Liu and Y. Wang, J. Mater. Chem., 2011, 21, 3568-3570.

49 Y. Qian, S. Li, G. Zhang, Q. Wang, S. Wang, H. Xu, C. Li, Y. Li and G. Yang, J. Phys. Chem. B, 2007, 111, 5861-5868.

50 H. Lin, X. Chang, D. Yan, W.-H. Fang and G. Cui, Chem. Sci., 2017, 8, 2086-2090.

51 V. S. Padalkar and S. Seki, Chem. Soc. Rev., 2016, 45, 169-202.

52 H. Wen, Q. Huang, X.-F. Yang and H. Li, Chem. Commun., 2013, 49, 4956-4958.

53 J. Cheng, D. Liu, W. Li, L. Bao and K. Han, J. Phys. Chem. C, 2015, 119, 4242-4251. 